\title{
Older Population
}

National Cancer Institute

\section{Source}

National Cancer Institute. Older Population. NCI Thesaurus. Code C18908.

The population of retired people, generally over 62 